\title{
Die Natur der Gefahr. Zur Geschichte der Überschwemmungsversicherung in Deutschland und den USA
}

\author{
Uwe Luebken
}

\begin{abstract}
In response to ever growing material damages caused by floods over the last two centuries, societies have sought techniques and methods to prevent, minimize or at least mitigate the physical and social havoc wreaked by such events. It was not until the middle of the nineteenth century, however, that insurance was added to the toolbox of flood management practices. The history of flood insurance is, by and large, a history of failure, at least as far as private insurance companies are concerned. Due to the lack of statistics, an accumulation of "bad risks" (adverse selection), and hydro-geographical factors, the insurance industry has to this day not been able to provide broad financial protection against flood hazards. Theoretically, insurance is an innovative way of dealing with contingency by spreading risk in time and space. In the case of flood insurance, however, nature's "behaviour" has turned out to be not simply less predictable than expected, it is literally unaccountable. Thus, in countries where flood insurance is available today, the government is usually involved - either as a provider of direct or indirect subsidies or as the actual insurer.
\end{abstract}

Keywords: Naturkatastrophen; Risiko; Versicherung; Überschwemmung; National Flood Insurance Program

Es soll die perfekte Abwechslung zum öden Büro- und Großstadtalltag werden, als die vier Protagonisten in John Boormans Hollywood-Klassiker Deliverance (1972) zu einem Wochenendtrip in die Berge Georgias aufbrechen, um mit ihren Kanus den (fiktiven) Cahulawassee River hinab zu fahren. Für die Geschäftsleute aus Atlanta ist dies die letzte Chance, den wilden, unregulierten und mit Stromschnellen übersäten Fluss zu bezwingen, denn die Arbeiten zum Bau eines Dammes laufen bereits auf Hochtouren und in wenigen Wochen wird ein riesiger Stausee die gesamte Gegend unter sich begraben. Am Fluss angekommen äußert Lewis Medlock (Burt Reynolds), der draufgängerische und Outdoorerfahrene Anführer des Quartetts, Zweifel an der Eignung des korpulenten Bobby (Ned Beatty). Ed (Jon Voight) versichert ihm jedoch, dass Bobby in seiner Branche, dem Versicherungsgeschäft, einer der besten sei, worauf hin Lewis lakonisch bemerkt: „Insurance? I've never been insured in my life. I don't believe in insurance. There's no risk.“

Wer sich also versichert, so mag man Lewis' Kommentar interpretieren, lebt risikoarm, und eine der letzten Möglichkeiten, dieser „Versicherungsmentalität“ zu entkommen, besteht darin, die Herausforderungen der ungezähmten Natur anzunehmen. Die von Boorman konstruierte Opposition von rationalem, bürokratischem Risikomanagement und natürlichen Prozessen, die diesem Kontrollapparat zuwider laufen, ist paradigmatisch für gängige Vorstellungen vom Verhältnis zwischen Natur und Gesellschaft in der Moderne. Schaut man aber etwas genauer hin, dann zeigt sich, dass die Auseinandersetzung 
mit der Dynamik von Flüssen und Meeren für die Versicherungsbranche ein ebenso großes Abenteuer war wie der Kanutrip für die vier Männer aus Georgia. ${ }^{1}$

\section{„Möglichkeitsbändigungen“}

Versicherungen sind ein innovatives Instrument zur Steuerung von Risiken und Unsicherheit (Bonß 1995). Die Aussicht auf finanzielle Kompensation nach Eintritt eines Schadensereignisses erzeugt Planungssicherheit und setzt Kapital und Energien frei, die ansonsten für die individuelle und kollektive Schadensbewältigung und Vorsorge hätten aufgewendet werden müssen. Das Versicherungsprinzip war jedoch lange Zeit alles andere als selbstverständlich. Die Vorstellung, sich durch die Zahlung eines Preises, der Prämie, gegen die ökonomischen Folgen eines zufälligen Schicksalsschlages abzusichern, galt über Jahrhunderte als sündhaft (Arps 1965, 16). Dies traf in besonderem Maße für die Lebensversicherung zu, denn den Körper eines freien Menschen mit einem monetären Wert abzuschätzen, widersprach sowohl dem römischen Recht als auch der christlichen Moral (Rotman 1979).

Mit dem Abklingen religiöser Deutungshoheit war die Zukunft jedoch nicht mehr prädestiniert, sondern grundsätzlich offen, und damit stieg auch der Bedarf an „Technologien des Risikos" (Ewald 1991, 198). Durch die Sozialisierung individueller Risiken konnten Versicherungen immer mehr Unsicherheiten des gesellschaftlichen Lebens statistisch bewältigen und damit finanziell in den Griff bekommen - vom plötzlichen Tod über den Verlust des Arbeitsplatzes bis hin zum Autounfall. ${ }^{2}$ In der modernen Gesellschaft ist die Versicherung ein zentrales Element der „Möglichkeitsbändigung“ (Makropoulos 1990); für manche stellen die Organisationsprinzipien der Versicherung sogar eine neue Form des Gesellschaftsvertrages, eine „Versicherungsgesellschaft“ dar (Ewald 1989).

Auch im Bereich der Absicherung gegen Naturgefahren haben Versicherungen Einzug gehalten, allerdings in sehr divergierendem Maße und mit sehr unterschiedlichem Erfolg. Während die Versicherungsbranche sehr erfolgreich mit Feuer- und Hagelschäden umzugehen gelernt hat und, mit Abstrichen, auch die Sturm- und Erdbebengefahr kalkulieren kann (Oberholzner 2006, Mohr 1963), stellen Überschwemmungen nach wie vor eine immense und zum großen Teil unbewältigte Herausforderung dar. Gerade die Auslotung dieser Grenzen der Versicherbarkeit macht die Geschichte der Überschwemmungsversicherung aber interessant.

\section{Der „Unwetter-Ausschuss“}

Jahrhundertelang waren private Wohltätigkeit und staatliche Unterstützung die einzigen Rettungsanker für die von Überschwemmungen Betroffenen. Erst gegen Mitte des

1 Es fand sich im Übrigen keine Versicherungsgesellschaft, die willens gewesen wäre, die extrem gefährlichen Dreharbeiten zu versichern, so dass die Schauspieler, die alle stunts selber ausführten, unversichert waren.

2 Die Praxis der Versicherung dient dabei nicht in erster Linie der Entschädigung oder der Kompensation für erlittenen Schaden, sondern dem Management von Unsicherheit (Ewald 1991). Oft wird vergessen, dass Versicherungen selbst auch ein Risiko darstellen. Der finanziellen Absicherung der versicherten Person durch die Versicherungsgesellschaften steht die „Gefahr“ des Nichteintritts des Schadensfalles gegenüber (Luhmann 1996). 
19. Jahrhunderts wurde versucht, das Katastrophenmanagement um ein neues Instrument zu erweitern: die Hochwasserversicherung. Die erste europäische Police wurde 1845 von der Azienda Assicuratrice in Triest ausgegeben. Die Unternehmung der österreichischungarischen Gesellschaft hatte nicht lange Bestand, doch andere folgten bald diesem Beispiel, insbesondere nachdem 1865 schwere Überschwemmungen große Teile Frankreichs getroffen hatten (Rommel 1950, 24f.). In Deutschland begannen ernsthafte Bestrebungen, eine Versicherung gegen Überschwemmungen zu etablieren, erst gegen Ende des 19. Jahrhunderts. Ausgangspunkt dafür war ausgerechnet ein Sturm.

Am 7. August 1898 richtete ein Orkan in der Kölner Region verheerende Schäden an. Betroffen waren nicht nur Privathäuser, Kirchen und infrastrukturelle Einrichtungen, sondern auch und gerade industrielle Anlagen. Die Schäden waren so verheerend, dass das Interesse in den Kreisen der Großindustrie nach finanzieller Absicherung gegenüber solchen Ereignissen deutlich wuchs. Aus diesem Grund fiel die Anregung des Vereins der Kölner Industriellen, eine Unwetterversicherung einzuführen, ,in den großgewerblichen Kreisen auf fruchtbaren Boden“ (Verein der Industriellen 1899). ${ }^{3}$ Am 1. März 1899 konstituierte sich in Berlin ein „Unwetter-Ausschuss“, der sich nach langen Diskussionen auf den Entwurf einer Versicherung verständigte, die Schäden durch „Muren, Hagelschlag, Windbruch und Wolkenbruch" abdecken sollte. Wasserschäden durch andere Überschwemmungen - und hiermit waren primär Flussüberschwemmungen gemeint - waren jedoch vorerst „nicht in diesen Begriff hineinzuziehen“, um den Gegenstandsbereich nicht zu sehr auszudehnen und um die Chancen auf Realisierung zu erhöhen (UnwetterAusschuss 1899).

Als potenzielle Träger der neuen Versicherung hatte man vor allem die privaten und öffentlichen Feuerversicherungsgesellschaften im Visier, die nicht nur Erfahrung mit der Abdeckung von zumindest teilweise natürlichen Gefahren, sondern auch den notwendigen Kapitalstock und reichliche finanzielle Reserven hatten - Vorteile, die gerade in der Gründungsphase neuer Versicherungen oft über Erfolg oder Ruin entscheiden konnten. Zudem verfügten sie in der Regel über einen ausgebauten Verwaltungsapparat sowie ein Netz an Agenten und andere Distributionsmethoden, die die Vermarktung in der Fläche erheblich erleichterten.

Die Adressaten wollten allerdings den „Sprung ins Dunkle“, den eine Unwetterversicherung ihrer Ansicht nach wegen des völligen Mangels an statistischem Material bedeutete, nicht wagen (Verband 1898). Noch ablehnender war das Urteil der Fachpresse. So bezeichneten die Annalen des gesamten Versicherungswesens (1899a) die Vorstellungen des Unwetterausschusses als einen „sonderbaren Beschluss“. Die staatlichen Feuerversicherer würden aus freien Stücken keinesfalls ,das Experiment wagen, weil sie sicher daran zu Grunde gehen würden“. Den privaten Versicherungsgesellschaften sollte die Regierung sogar verbieten, „sich auf ein so gefährliches, völlig unberechenbares Geschäft einzulassen, da das nur die Sicherheit der gegen Feuer- und Hagelschaden Versicherten gefährden könnte“ (Annalen des gesamten Versicherungswesens 1899a). ${ }^{4}$

3 Zu Beginn der 1920er Jahre versicherten in Deutschland lediglich die Colonia, die Kölnische Feuer- und Unfall-Versicherungs-Aktien-Gesellschaft und die Stuttgart-Berliner Versicherungs-Aktiengesellschaft Sturmschäden und dies auch nur für ,feststehende Gebäude“ und deren Inhalt. Für Feldfrüchte und Wälder bestand kein Versicherungsschutz (Reichsaufsichtsamt 1920).

4 In einer späteren Ausgabe derselben Zeitschrift (1899b) ist von dem „Unsinnigen ihres [des Industriellenverbandes] Verlangens“ die Rede. Weiter heißt es: „Die privaten Feuerversicherungs-Gesellschaften wür- 
Auf ebenso wenig Gegenliebe stieß die Überschwemmungsversicherung, die, obwohl vom Unwetterausschuss ausgeschlossen, parallel zur Unwetterversicherung breit diskutiert wurde. Ein Grund hierfür lag in der Forderung agrarischer Kreise nach der Einbeziehung von Hochwasserschäden, ,denen die Landwirtschaft seit 20-30 Jahren in früher nicht gekanntem Umfange ausgesetzt ist" (Annalen des gesamten Versicherungswesens 1899b). Eine Überschwemmungsversicherung wurde vom Preußischen Versicherungsbeirat zwar nicht grundsätzlich ausgeschlossen, jedoch als „,gegenwärtig nicht durchführbar“ bewertet, weil sie „Zweien, versicherungstechnisch unentbehrlichen Fundamentalgrundsätzen“" widerspreche (Annalen des Deutschen Reiches 1899). Neben dem Mangel an statistischem Material bezog sich der Beirat hier auf das Problem der adversen Selektion, also einer „negativen Risikoauslese“ (Kalenberg 1998, 8f.). Während die Gefährdung durch Feuer oder Sturm potenziell alle Hausbesitzer betrifft, ist die Überschwemmungsgefahr räumlich relativ deutlich einzugrenzen. Das Wissen der potenziellen Versicherungsnehmer um die Gefährdung bzw. Nicht-Gefährdung hat zur Folge, dass sich vorwiegend „schlechte Risiken“ in der Versicherung wieder finden werden, ein Risikoausgleich im Schadensfall also nur sehr begrenzt möglich ist.

Das statistische Informationsdefizit und das ungelöste Problem der adversen Selektion, aber auch Uneinigkeit unter den Ausschussmitgliedern über die Höhe der Beiträge, mangelnder Konsens in Bezug auf die Definition von „Unwetter“ sowie die Begrenzung der zu versichernden Gegenstände führten dazu, dass sich knapp ein Jahr nach der Initiative der Kölner Industriellen wenig bewegt hatte (Deutsche Versicherungs-Zeitung 1900). In einer Hinsicht zeitigte die Initiative allerdings Erfolg. Das Preußische Statistische Landesamt kam in der Tat der Anregung nach, statistische Erhebungen über Unwetterschäden jeglicher Art „für einen möglichst großen Zeitraum rückwärts“ vorzunehmen. Diese Daten, so hatten die Kölner Industriellen gefordert, sollten auch Überschwemmungen beinhalten, da „,doch ein großes volks- und staatswirtschaftliches Interesse an der Möglichkeit, Ueberschwemmungsgefahren versichern zu können“, bestehe (Verein der Industriellen 1899). Von 1900 bis 1916 wurden statistische Untersuchungen über Hochwasser und Überschwemmungsschäden angefertigt und in der Preußischen Statistik veröffentlicht. Die Auswertung der Daten wurde jedoch im Laufe des Krieges zunehmend schwieriger. Ab 1917 fanden überhaupt keine Erhebungen mehr statt, da solche Aktivitäten nach einem Erlass des Innenministers „während des Krieges als weniger wichtig einzustellen“ waren (Quante 1926).

\section{Das Schweizer Modell: Die obligatorische Elementarschadenversicherung}

Nach Kriegsende wurden die Pläne zur Schaffung einer Unwetter- und Überschwemmungsversicherung aus mehreren Gründen wieder aus den Schubladen geholt. Zum einen erhöhten verschiedene Naturkatastrophen auf der ganzen Welt, wie das gewaltige Erdbeben in Tokio 1923 oder der Hurrikan in Florida 1927, die Sensibilität für das zerstörerische Potenzial von Naturkräften (Hutchinson 2000, 4f.). Zum anderen wurde Deutsch-

den sträflich leichtsinnig handeln, wollten sie durch Übernahme des Unwetterrisikos die Sicherheit ihrer Versicherten gefährden. Halten die Industriellen und die Landwirte die Versicherung gegen Unwetterschäden wirklich für so notwendig, wie sie jetzt thun, so mögen sie doch das Aktienkapital oder den Garantiefonds für eine eigene Gesellschaft aufbringen.“ 
land, und insbesondere das Rheinland, in den 1920er Jahren mehrfach von Überschwemmungen heimgesucht, so dass das Thema in der Öffentlichkeit, der Presse und im Parlament aktuell blieb. Im März 1926 plädierte Volkswirtschaftsrat Quante vom Preußischen Statistischen Landesamt für eine Wiederaufnahme der alljährlichen Feststellung von Überschwemmungsschäden. Die Erfahrung des laufenden Jahres - und damit meinte der Beamte das Winterhochwasser 1925/26 - zeige, ,dass eine fortlaufende, nach einheitlichen Grundsätzen aufgebaute Statistik der Überschwemmungsschäden durchaus notwendig ist" (Quante 1926).

Interessant an Quantes Ausführungen ist der Wandel in der Risikoaufteilung zwischen Staat, privater Fürsorge und Versicherungen im Vergleich zur Vorkriegszeit. Noch 1900 hatte das Preußische Statistische Bureau eine allgemeine Unwetterversicherung auch mit dem Hinweis darauf abgelehnt, dass der ,,reicher gewordene und besser geordnete Staat sowie die wohlhabendere und ihrer Pflichten bewusste Bevölkerung (...) heutzutage schneller und mit beträchtlicheren Mitteln als vordem den Leuten bei[springen], welche von derartigen Prüfungen heimgesucht sind." Leistungsfähigkeit von Staat und Gesellschaft repräsentierten also die eigentliche Versicherung für Flut- und Unwettergefährdete. Nach dem Krieg hatte sich die Situation jedoch erheblich gewandelt. Der stetige Anstieg von Beihilfezahlungen und Notstandskrediten, insbesondere nach der Serie von Überschwemmungen in den 1920er Jahren, ging mehr und mehr zu Lasten des Staates, der durch die generelle wirtschaftliche Lage ohnehin schon stark belastet war. Sollte es gelingen, die Risikovorsorge den potenziell Betroffenen in Form einer Unwetter- oder gar einer Überschwemmungsversicherung aufzubürden, so würde dies zu einer erheblichen Entlastung des Staatshaushaltes führen. Dies hatte die Erfahrung mit der Versicherung gegen andere Naturgefahren deutlich gemacht. Die Hagelversicherung z.B. hatte seit der Währungsstabilisierung einen beachtlichen Aufschwung genommen. Weil somit im ganzen Land Versicherungsschutz erhältlich sei, müssten bei Hagelschäden keine Notstandsaktionen mehr eingeleitet werden. Die Existenz privatwirtschaftlichen Versicherungsschutzes diente also als Rechtfertigung für einen Rückzug des Staates aus der Katastrophenhilfe. Die Implikationen für die Staatsfinanzen waren offensichtlich: „Wenn die im Gange befindlichen Erhebungen die Einführung einer Unwetterschädenversicherung als möglich erscheinen lassen sollten“, schrieb ein Mitarbeiter des Preußischen Innenministeriums, ,würde das allerdings für den Staat eine ganz wesentliche Entlastung in der Bereitstellung von Notstandsmitteln bedeuten“ (Preußisches Innenministerium 1930).

Die neue Dringlichkeit manifestierte sich auch im Interesse an den Erfahrungen anderer Länder. Ermittlungen des Auswärtigen Amtes ergaben, dass, von einigen unbedeutenden Versuchen abgesehen, eine Hochwasserpolice bisher nur in der Schweiz und in Österreich angeboten worden war. ${ }^{5}$ Die österreichische Gesellschaft Providentia hatte ihre Tätigkeit unmittelbar vor Kriegsbeginn aufgenommen. Während ihrer vierjährigen Existenz (1914-1917) verkaufte das Unternehmen 53 Policen und nahm etwa 100.000 Kronen an Prämienzahlungen ein. Die Versicherungsnehmer meldeten eine Gesamtzahl von 20 Schäden, die zu Zahlungen von 190.000 Kronen für die Wiener Gesellschaft führten. Die Schweizerische National-Versicherungs-Gesellschaft hatte zwar nicht so stark unter aktuellen Schäden zu leiden wie ihr österreichisches Pendant, der Umfang der helvetischen

5 Rommel (1950, 24-26, 42-44) benennt darüber hinaus noch frühe Versuche in Dänemark, Bulgarien. Norwegen und Japan. 
Unternehmung blieb jedoch gering und unprofitabel. Nach kurzer Zeit gaben beide Unternehmen ihre Flutversicherungsaktivitäten wieder auf (Münchner Rück 1927).

In der Schweiz wurde jedoch in der Folgezeit ein Modell entwickelt, das bis heute in der Eidgenossenschaft Anwendung findet, und das Ende der 1920er Jahre in Berlin mit großem Interesse zur Kenntnis genommen wurde: Die Inkorporierung der Überschwemmungsversicherung in eine obligatorische Elementarschadenversicherung. Im August 1927 reisten Vertreter der deutschen Versicherungswirtschaft und mehrere Regierungsbeamte nach Bern, um sich von Hermann Lanz-Stauffer, dem Direktor des Interkantonalen Rückversicherungsverbandes, über die bisherigen Erfahrungen mit dieser Versicherung unterrichten zu lassen. Zwei Charakteristika zeichneten das Schweizer Modell aus: Zum einen war der Abschluss einer solchen Versicherung Pflicht für alle Hausbesitzer, zum anderen beinhaltete sie Schutz vor mehreren Elementargefahren gleichzeitig, also nicht nur Überschwemmungen, sondern auch Sturm, Lawinen oder Wolkenbruch. ${ }^{6}$ Die von den kantonalen Feuerversicherungsanstalten getragene Elementarschadenversicherung hatte mehrere Vorteile. Die aufwändige und stets umstrittene Abgrenzung verschiedener natürlicher Schadensursachen war nun hinfällig, das Obligatorium sorgte für hohe und regelmäßige Prämienzahlungen, und durch den umfassenden Schutz konnte auch die Ausweitung der Versicherung auf nicht von Hochwasser betroffene Hausbesitzer gerechtfertigt werden. Schließlich machte diese Quersubventionierung die Versicherung gegen Überschwemmungen überhaupt erst möglich (Preußisches Innenministerium 1927a, LanzStauffer/Rommel 1936, Fischer 2004).

In Preußen fiel das Schweizer Modell auf fruchtbaren Boden und führte immerhin zu einem Gesetzentwurf über die „Einrichtung einer Hochwasser- und Unwetterschädenversicherung“. Wie in der Schweiz sollte die Hochwasserversicherung in Preußen Zwangscharakter haben und auf die „breiteste Basis“ gestellt werden. Das hieß, dass sämtliche Grundbesitzer die Prämie zu zahlen hätten und dass Schutz vor allen Elementarschäden bis auf Hagel und Erdbeben gewährt würde. Zwar wurden Bedenken laut gegen die Einbeziehung von Sturmfluten. Der entsprechende Passus wurde aber beibehalten, „da bisher Notstandsaktionen bei Schäden durch Springfluten kaum notwendig gewesen sind und Aufnahme der Meeresschäden politisch erwünscht erscheint“ (Preußisches Innenministerium 1927a).

Der wichtigste Unterschied zum Schweizer Modell bestand darin, dass landwirtschaftliche Schäden „angesichts der deutschen Verhältnisse“ unbedingt einbezogen werden sollten. Dies sollte vor allem die gegenwärtige Ernte betreffen, nicht aber mittelbare Schäden wie etwa durch Verschlammung der Felder. Ebensowenig sollte das Vieh versichert sein. Die Beitragshöhe sollte sich generell nach der Grundvermögenssteuer richten; wer sein Land aber in den „gebietsweise zusammenzufassenden Hochwassergebieten“ hatte, musste mit einem Zuschlag zum Grundbetrag rechnen. Die Prämie sollte zunächst relativ hoch angesetzt werden, um dann später, nach dem Abgleich mit den tatsächlichen Hochwasserschäden, wieder herunter gesetzt werden zu können. Getragen werden sollte die Versicherung von einer eigenen öffentlich-rechtlichen Hochwasserversicherungsanstalt, analog zu den existierenden öffentlichen Feuerversicherungsgesellschaften und un-

6 Ausgenommen waren Erdbeben und „vulkanische Schädigungen“. Die Versicherung existierte zu dieser Zeit bereits in den Kantonen Waadt und Appenzell und war in Bern, Neuenburg, St. Gallen und BaselLand im Entstehen begriffen (Preußisches Innenministerium 1927a, Johner 1958, 7f.). 
ter der Aufsicht des Preußischen Innenministeriums. Die Verwaltung der neuen Gesellschaft sollte jedoch vom Feuerverband übernommen werden (Preußisches Innenministerium $1927 \mathrm{a}$ und $\mathrm{b}$ ).

Im Gegensatz zu der ersten Initiative in den 1890er Jahren gingen die Bestrebungen zur Einführung einer Hochwasserversicherung nun nicht mehr von privatwirtschaftlichen Kreisen, sondern vom Staat aus. Die private Versicherungswirtschaft blieb zwar weiterhin skeptisch, wenn auch nicht mehr so polemisch argumentierend wie gut 35 Jahre zuvor, doch die öffentlichen Feuerversicherer waren aufgeschlossener als im Kaiserreich, und das Schweizer Modell schien die strukturellen Probleme der Überschwemmungsversicherung lösen zu können. Auch Mangel an statistischem Material war nun aufgrund der Arbeiten des Statistischen Landesamtes kein Hinderungsgrund mehr. Die Etablierung der Versicherung scheiterte jedoch an den ökonomischen und politischen Verwerfungen der späten Weimarer Republik, ,weil aller Voraussicht nach mit der Durchführung dieser Versicherung eine äußerst empfindliche neue Mehrbelastung der Wirtschaft verbunden sein wird“, wie ein Mitarbeiter des Preußischen Ministeriums für Landwirtschaft, Domänen und Forsten im Januar 1931 festhielt. Im Schadensfall wären die Ansprüche auf Entschädigung zahlreicher und höher als bisher. Zudem würden etliche Betriebe mit Mehrkosten belastet, die die neue Versicherung kaum jemals in Anspruch nehmen würden. Die Hauptlast wäre von der Wirtschaft selbst zu tragen, während der öffentlichen Hand nur ein verhältnismäßig geringer Anteil zukäme. Eine derartige Lösung war aber ,unter den heutigen wirtschaftlichen Verhältnissen nicht tragbar und nicht erreichbar. [...] Vielmehr wird sich der Staat vorläufig der Notwendigkeit nicht entziehen können, bei Katastrophen, die gewisse Grenzen überschreiten, in der Hauptsache von sich aus helfend und lindernd einzugreifen“ (Arnoldi 1931). Das, was eigentlich das Ziel war, nämlich die Abwälzung der Risikoübernahme vom Staat hin zu den Privathaushalten und den Unternehmen, wurde nun als kontraproduktiv angesehen.

\section{Die Entwicklung in Deutschland nach dem Zweiten Weltkrieg}

In der Nachkriegszeit setzte sich die Geschichte des Scheiterns der Überschwemmungsversicherung nahtlos fort. Zwar gab es in der Bundesrepublik immer wieder entsprechende Initiativen, wie etwa vom Rheingaukreis nach dem schweren Hochwasser am Mittelrhein 1956. Diese stießen aber regelmäßig auf Ablehnung seitens der Versicherungsbranche (Landrat 1957). Auch die staatlichen Aufsichtsorgane zeigten sich wenig aufgeschlossen. Das Bundesaufsichtsamt für Versicherungswesen (BAV) verwies auf das Problem der adversen Selektion und hielt 1960 fest (eine Botschaft, die das Amt in den Jahren 1966 und 1979 wiederholen sollte), dass Hochwasserschäden auf privatwirtschaftlicher Basis nicht zu versichern seien, da ,sich an einer derartigen Versicherung nur Versicherungsnehmer beteiligen würden, deren zu versichernde Sachwerte in einem vom Hochwasser bzw. einer Überschwemmung bedrohten Gebiet liegen“" (zit. nach Kalenberg 1998, 3).

Im Jahr 1990 setzte jedoch ein erstaunlicher Sinneswandel ein, als die über ein Jahrhundert eisern vertretene Auffassung der deutschen Versicherungswirtschaft und des BAV sowie seiner Vorgänger, dass Elementargefahren mit der Ausnahme von Sturm und Hagel nicht versicherbar seien, aufgegeben wurde. Ausschlaggebend für diese „Revolution" war aber weniger die plötzliche Einsicht in die Machbarkeit einer solchen Versicherung als vielmehr die Furcht, ausländische Versicherungsgesellschaften könnten von den jeweiligen Staaten subventionierte Angebote von Elementarschadenversicherungen - vor 
allem gegen die Überschwemmungs- und Erdbebengefahren - als Köder für das Eindringen in den deutschen Markt nutzen und somit die Wettbewerbsfähigkeit deutscher Unternehmen unterminieren (Kalenberg 1998, 4f.). So verwundert es auch kaum, dass die deutsche Versicherungswirtschaft in der Folgezeit Hochwasserpolicen zwar angeboten, jedoch nicht offensiv vermarktet hat. Der Bestandsaufbau erfolgte dementsprechend nur sehr langsam und unter Vermeidung von Risikoakkumulationen (Böhm 1998, 108f.). Eine Ausnahmesituation bestand in den neuen Bundesländern, in denen die private Versicherungswirtschaft viele Verträge aus DDR-Zeiten fortgeführt hatte, von denen 60 bis 70 Prozent das Hochwasserrisiko abdeckten. Diese Konstellation sollte zu enormen Zahlungen und umfangreichen Vertragskündigungen nach der Oderflut 1997 und dem „Jahrhunderthochwasser" 2002 an der Elbe führen (Schwarze/Wagner 2004).

Da auf privater Basis kein ausreichender finanzieller Schutz vor Überschwemmungen zu haben war, wurden immer wieder Rufe nach einer staatlichen oder staatlich subventionierten Pflichtversicherung laut. Nur ein einziges Bundesland, Baden-Württemberg, war jedoch dem Schweizer Modell gefolgt, indem es 1960 eine obligatorische Elementarschadenversicherung für alle Hausbesitzer einführte, die auch Schäden durch Überschwemmungen abdeckte (Kalenberg 1998, 110). Dieser „Sonderweg“" wurde allerdings durch die Wettbewerbspolitik der Europäischen Union beendet. Mit dem EU-weiten Verbot von Versicherungsmonopolen ab dem 1. Juli 1994 musste auch die baden-württembergische Pflichtversicherung ihre Tätigkeiten einstellen. Zwar waren Pflichtversicherungen weiterhin möglich, nur durften diese nicht mehr von staatlichen Monopolgesellschaften betrieben werden (Böhm, 107). Die bayerische Regierung machte nach dem desaströsen Pfingsthochwasser 1999 einen neuen Anlauf und schlug eine Versicherungspflicht gegen Elementarschäden vor; diese war jedoch relativ schnell wieder vom Tisch. Im Gegensatz zur privatwirtschaftlichen Versicherung würde eine staatliche Pflichtversicherung ,eine durch den Bestand quersubventionierte Versicherung" bedeuten, stellte Horst Kriebisch, Direktor des Gesamtverbandes der deutschen Versicherungswirtschaft, fest. Damit würde aber „ein evtl. vorhandenes risikoorientiertes Verhalten der Bürger abgeschafft, zumindest jedoch reduziert“ und die Versicherung ,in den hochexponierten Zonen zu einer Schadenerzeugungsmaschine“" (Kriebisch 2001, 128). ${ }^{7}$

Die Versicherungswirtschaft ist seitdem bei ihrer Ablehnung der Pflichtversicherung geblieben und setzt im privaten Bereich auf eine geographische Kartierung der Risiken, die es ermöglichen soll, die Prämien in Einklang mit den tatsächlichen Risiken zu bringen. Abgesehen davon, dass solche Zonierungen im Einzelfall höchst umstritten sein können, ist jedoch fraglich, ob die Gefährdeten die hohen Prämien zu zahlen bereit sein werden. Dass Privatversicherungen zur Zeit keine Alternative zu staatlichen Hilfsleistungen darstellen, wurde zuletzt im Februar 2008 deutlich, als Ministerpräsident Beckstein der bayerischen Versicherungskammer erklärte, er halte es für „sehr wichtig, nochmals die Möglichkeiten zur Einführung einer verpflichtenden Elementarschadensversicherung zu untersuchen." Während sich in Deutschland also weder die Privatversicherer noch der Staat zur Einführung einer funktionierenden Überschwemmungsversicherung durchringen konnten, beschritten die USA gewissermaßen einen „dritten Weg“, der im Folgenden geschildert werden soll.

7 Für das französische CatNat System s. Schwarze/Wagner 2004, 165f., Kalenberg 1999, 62-71. 


\section{Flood Insurance in den USA}

Für die USA wurden, wie für andere industrialisierte Länder auch, Hochwasser mehr und mehr zu einem Problem, seit ab etwa der Mitte des 19. Jahrhunderts die „Invasion“ der Überschwemmungsgebiete begann, d.h. die Errichtung von immer mehr Straßen, Eisenbahnlinien, Industrieanlagen, Warenhäusern, Wohngebieten etc. im natürlichen Ausdehnungsbereich der Flüsse (Luebken 2007). Mehrere verheerende Überschwemmungen am Mississippi, in Neuengland und am Ohio River im ersten Drittel des 20. Jahrhunderts hatten nicht nur für gesellschaftliche Tragödien gesorgt (Barry 1997), sondern auch die zunehmende finanzielle Belastung für staatliche Organe auf allen Ebenen deutlich vor Augen geführt. Die Antwort auf diese Herausforderung wurde jedoch nach wie vor primär im baulichen Hochwasserschutz gesucht, maßgeblich beeinflusst von der „levee only“-Doktrin des Army Corps of Engineers.

Mit der Verabschiedung des Flood Control Act 1936 wurde der Hochwasserschutz explizit in den Aufgabenbereich des Bundes gelegt, der diesen bis dahin nur sporadisch betrieben hatte. Doch trotz der Errichtung hunderter neuer Deiche, Dämme, Schutzmauern und einiger Rückhaltebecken stiegen die volkswirtschaftlichen Schäden durch Überschwemmungen stetig an, und dies sogar schneller als die Ausgaben für den Hochwasserschutz. Mit jeder neuen Flut wurde deutlicher, dass dem Hochwasserproblem nicht allein durch Beton und aufgeschüttete Erde beizukommen war (HUD 1966, 44).

Auch im Bereich der Katastrophenhilfe stiegen die finanziellen Belastungen drastisch an, nachdem die US-Bundesregierung mit der Verabschiedung des Disaster Relief Act im Jahr 1950 auch hier systematisch (und nicht mehr nur im Einzelfall) involviert war (Rozario 2007, 150-55, Platt 1999, 70). „Whenever a major disaster strikes anywhere in the United States today", hielt das Ministerium für Wohnungsbau und Stadtplanung 1966 fest, "substantial public help and relief are available" (HUD 1966, 54). Diese Verfügbarkeit von Hilfsmitteln erhöhte aber nicht eben das Risikobewusstsein von Unternehmen und Hausbesitzern in den Überschwemmungsgebieten, und auch der bauliche Hochwasserschutz wirkte zum Teil kontraproduktiv, indem er ein falsches Gefühl von Sicherheit evozierte und „development“ hinter den Deichen förderte (Platt 1999, 70).

\section{Überschwemmungen und die privaten Versicherer in den USA}

Die Kombination aus ineffektivem Hochwasserschutz und steigenden Hilfszahlungen erzeugte massiven Handlungsdruck. Seit den frühen 1950er Jahren kursierten daher in den USA immer wieder Pläne und Gedankenspiele zur Errichtung einer Überschwemmungsversicherung (Langbein 1953, Grutzner 1955), nicht zuletzt beeinflusst durch die Ergebnisse einer Forschergruppe um den Chicagoer Geographen Gilbert F. White, die einen umfassenderen Ansatz zur Lösung des Hochwasserproblems forderte („floodplain management“; Platt 1999, 70, White, 1945). Gerade Versicherungen schienen ein ideales Instrument zu liefern, um den von der Flut Betroffenen Hilfe zukommen zu lassen und gleichzeitig eine verantwortliche Nutzung der Überschwemmungsgebiete zu fördern (Langbein 1953, 330, Rozario 2007, 170).

Wie in Europa ist auch in den USA die Geschichte der Überschwemmungsversicherung auf privatwirtschaftlicher Basis lange Zeit von gescheiterten Versuchen geprägt. In Cairo, Illinois, versanken 1899 selbst die Firmengebäude der zwei Jahre zuvor gegründeten Aktiengesellschaft, die gegen Hochwasser versicherte, in den Fluten des Mississippi (Rommel 1950, 25, Grutzner 1955). In den 1920ern gab es mehrere Unternehmen, die 
flood insurance anboten; deren Aktivitäten wurden jedoch jäh unterbrochen, als 1927 schwere Fluten zuerst das Mississippi-Tal und dann Neuengland trafen. ${ }^{8}$ Wie in Deutschland war auch in den USA die räumliche Konzentration von „schlechten Risiken“ das größte Problem, denn nur ein Bruchteil aller Haushalte in den USA war einer signifikanten Gefahr durch Überschwemmungen ausgesetzt, und innerhalb dieser kleinen Gruppe war die Risikobelastung äußerst ungleich verteilt. Weniger als ein Prozent dieser Haushalte trug die Hälfte aller Hochwasserrisiken, während sich das gesamte Restrisiko auf etwa zehn Prozent verteilte (HUD 1966, 28f.).

Problematisch war allerdings weniger die Tatsache, dass nur eine kleine Minderheit der Bevölkerung durch Überschwemmungen gefährdet war - dies gilt rückblickend schließlich für andere Naturgefahren in noch viel stärkerem Maße. Überschwemmungen markieren sogar ,by a wide margin, the most serious of the natural disaster hazards facing the people of the United States" (HUD 1966, 20f.). Tornados, Erdbeben oder Hagelschauer bedrohen aber immer ganze Regionen und erzeugen damit eine große Gefahrengemeinschaft, in denen sich bei Eintritt des Ereignisses niemand sicher sein kann, nicht zu den Opfern zu gehören. Bei Überschwemmungen dagegen lässt sich der Grad der Gefährdung nicht selten in Metern angeben. In Wareham, Massachussetts, betrug zum Beispiel die Entfernung von der Gefährdungszone A, in der Hochwasser im Durchschnitt alle fünf Jahre oder öfter auftraten, zur Gefährdungszone D, die im Durchschnitt nur alle 25 bis 50 Jahre überschwemmt wurde, weniger als 500 Fuß. In engen Flusstälern konnten diese Distanzen noch geringer sein. (HUD 1966, 28f.)

Neben der adversen Selektion machten aber auch andere strukturelle Probleme der Hochwasserversicherung zu schaffen. Zunächst brauchten die Gesellschaften, die dieses Risiko übernahmen, einen relativ großen Kapitalstock, da bei Überschwemmungen selten nur einzelne Versicherungsnehmer betroffen sind und die Schäden daher schnell kumulieren (Gerdes 1963, Kalenberg 1998, 6-8). Problematisch war selbst das Ausbleiben von Überschwemmungen. Mehrere „ereignisfreie“ Jahre ließen das Interesse an Versicherungsschutz drastisch sinken, wie die wenigen Gesellschaften fest stellen mussten, die flood insurance Policen in den USA angeboten hatten (American Insurance Association 1966). Bei der Feuerversicherung dagegen sorgten ,tausende von Bränden, die täglich mit ziemlich regelmässiger Vertheilung über grosse Gebiete stattfinden“ dafür, das Interesse wach zu halten. „Nur die Erkenntnis der steten Gefahr hat der weiten Verbreitung der Versicherung gegen Feuerschaden Vorschub geleistet", hielten die privaten deutschen Feuerversicherer schon Ende des neunzehnten Jahrhunderts fest (Verband 1898).

Wie in Deutschland machte die Versicherungswirtschaft in den USA unter Bezugnahme auf diese strukturellen Defizite mehrfach deutlich, dass Hochwasserschäden ihrer Ansicht nach nicht versicherbar waren, und dass von ihrer Seite keine Initiative zu erwarten sein würde (American Insurance Association 1966, HUD 1966, 64). Da die Kette von schweren Flutkatastrophen - sowohl an der Küste wie auch an den Flüssen - aber nicht abriss, richteten sich die Augen mehr und mehr auf den Staat.

8 Für das Ende des Flutversicherungsgeschäftes der Globe \& Rutgers Fire Insurance Company sowie der Hartford Fire Insurance Company vgl. Flood Insurance Halted, New York Times, 26. April 1927, und Suffering in Hartford Increased by Cold, ebd., 9. November 1927. 


\section{Das National Flood Insurance Program (NFIP)}

Erste zaghafte Versuche der Regierung Truman zur Einführung eines nationalen Versicherungsfonds nach Rekordfluten in Kansas und Missouri im Sommer 1951 scheiterten (Langbein 1953, 328), doch eine Reihe von weiteren Flutkatastrophen verlieh den Argumenten für die Einführung einer Überschwemmungsversicherung Dringlichkeit (Gerdes 1963). 1955 waren es Hurrikane im Nordosten der USA und Überschwemmungen in Kalifornien, die das Thema wieder aufs Tapet brachten. Nach Anfertigung eines umfangreichen Berichts durch die Mitarbeiter des United States Senate Banking and Currency Committee (1956) wurde im August 1956 der Federal Flood Insurance Act verabschiedet, der ein von der Bundesregierung subventioniertes Versicherungssystem vorsah, das allerdings von privaten Gesellschaften ausgeführt werden sollte. Die Finanzierung durch den Kongress blieb jedoch aufgrund von Zweifeln an der Durchführbarkeit aus. 1957 wurde die eigens gegründete Federal Flood Indemnity Administration wieder aufgelöst (HUD 1966, 65, King 2005, 6f.). Dass es gut zehn Jahre später dennoch zu einem staatlichen Versicherungsprogramm kam, lag vor allem an Hurrikan Betsy, der im September 1965 in der Golfregion schwere Verwüstungen hinterließ. Der noch im selben Jahr verabschiedete Southeast Hurricane Disaster Relief Act sah nicht nur finanzielle Hilfen für die Flutopfer vor, sondern autorisierte auch eine Möglichkeitsstudie für ein nationales Versicherungsprogramm. Die zwei hieraus resultierenden, umfangreichen Berichte (HUD 1966, United States Task Force on Federal Flood Control Policy 1966) lieferten die Basis für den National Flood Insurance Act, der 1968 den Kongress passierte.

Der Grundgedanke des NFIP ist von seiner Gründung an der gleiche geblieben, auch wenn die konkrete Ausgestaltung über die Jahrzehnte erheblichem Wandel unterlag. Städte und Gemeinden, die von der Federal Insurance Administration (FIA) als hochwassergefährdet eingestuft werden, können am Programm teilnehmen und ihren Einwohnern von der Bundesregierung subventionierte Policen anbieten, wenn sie dafür im Gegenzug in den betreffenden Gebieten Maßnahmen ergreifen, die die Hochwassergefahr verringern. 1969 begann die Kooperation zwischen der FIA und der National Flood Insurers Association (NFIA), einem Zusammenschluss von 89, später über 100, privaten Sachversicherern. Die NFIA übernahm die Verwaltung der Policen und die Schadensregulierung, während die Bundesregierung das Programm finanziell unterstützte und einen Großteil des Risikos trug. Um festzustellen, ob eine Gemeinde hochwassergefährdet war, erstellte die FIA flood hazard boundary maps, die den gefährdeten Bereich grob abgrenzten und veranlasste weitere hydrologische und topographische Studien zur genauen Berechnung der Prämien. Das Ergebnis dieser Arbeiten, die mehrere Jahre andauern konnten, war eine Flood Insurance Rate Map (FIRM), die den Orten in der Gemeinde verschiedene Gefährdungsklassen und somit unterschiedliche Prämien zuwies (Power/Shows 1979, 62f.).

In den ersten Jahren war die Resonanz auf das Programm gering. Als 1972 ,tropical storm" Agnes Rekordhochwasser, vor allem in Pennsylvania, erzeugte, war die Überraschung groß, dass nur ein Bruchteil der Opfer versichert war. Als Gründe für dieses Defizit wurden der Mangel an Informationen über die individuellen Hochwasserrisiken und die trotz der Subventionen zu hohen Prämien angeführt (Platt 1999, 71, Power/Shows 1979, 64). Um die Zahl der Versicherten zu erhöhen, wurde 1973 der freiwillige Charakter des Programms stark eingeschränkt. Der Nachweis einer Überschwemmungsversicherung wurde zur Pflicht für Hausbesitzer, die von der Bundesregierung subventionierte Hypothekendarlehen erhalten wollten. Waren im Dezember 1973 lediglich Werte in der Höhe von 5,5 Milliarden Dollar versichert, so wuchs diese Zahl bis November 1977 auf 36 Milliarden (Power/Shows 1979, 61). Die Zahl der ausgegebenen Policen stieg von 
lediglich 95.000 zum Zeitpunkt von „Agnes“ auf 1,2 Millionen im Jahr 1977, während sich die Anzahl der teilnehmenden communities im gleichen Zeitraum von 2.200 auf 15.000 erhöhte (FEMA 2002).

\section{Probleme des NFIP}

Bei der Gründung des NFIP war geplant, das Programm sich selbst finanzieren zu lassen, d.h., dass der Ertrag aus den Prämien groß genug sein sollte, um die Zahlungen im Schadensfall zu decken. Sollten große Katastrophen die Finanzkraft des NFIP überfordern, konnte es einen Kredit beim amerikanischen Finanzministerium aufnehmen. Diese Konstruktion geriet aber immer wieder in Schieflage, so dass zwischen 1986 und 1997 ein Nettoverlust von 1,1 Milliarden Dollar auflief, der letztlich von den amerikanischen Steuerzahlern beglichen wurde. Auf der anderen Seite haben die Versicherungsnehmer im selben Zeitraum immerhin 6,4 Milliarden Dollar durch ihre Prämienzahlungen selbst aufgebracht, so dass nach Einschätzung Platts $(1999,72)$ das NFIP immer noch effektiver ist als staatliche Hilfszahlungen.

Ein Grund für die hohen Auszahlungen des NFIP waren „Repetitive Loss Properties“ (RLP). Im Gegensatz zur privaten Versicherungswirtschaft, die bei mehrfachen Schadensfällen eines Versicherungsnehmers in kurzer Zeit den Vertrag kündigen würde, hat das NFIP diese schlechten Risiken weiter versichert. Dies führte dazu, dass im Jahr 1998 über ein Viertel aller claims an das NFIP von RLPs kamen, von denen wiederum 37 Prozent drei mal oder noch häufiger geschädigt wurden. Von den Auszahlungen gingen sogar 40 Prozent an diese mehrfach von Fluten betroffenen Versicherten. Hierzu gehörten nicht nur Eigenheimbesitzer aus der Mittelschicht, sondern - und dies wurde natürlich in der Presse mit großem Interesse zur Kenntnis genommen - auch etliche Einrichtungen von nicht gerade mittellosen Amerikanern. In Long Island zum Beispiel erhielt eine Bar in einem unmittelbar am Wasser gelegenen Resort 1995 und 1996 zwei Zahlungen in Höhe von insgesamt 752.000 Dollar - die höchste Summe, die das NFIP jemals in Long Island ausgezahlt hat (Platt 1999, 73).

Auch die Beteiligung am NFIP ist trotz der mittlerweile 4,5 Millionen Policen eher enttäuschend. Dies liegt zum Teil daran, dass Verstöße gegen die Versicherungspflicht kaum geahndet wurden, so dass Hausbesitzer, die ihren Prämienzahlungen nicht nachkamen, zwar aus dem Programm fielen und ihren Versicherungsschutz verloren, aber keine Strafen fürchten mussten. Ein weiteres Reformgesetz 1994 nahm die Kreditgeber stärker in die Pflicht, doch „,non-compliance“ blieb ein gewichtiges Problem des NFIP (King 2005). Zudem unterlagen Darlehensgeber, die nicht vom Staat reguliert wurden, ebenso wenig der Versicherungspflicht wie Hausbesitzer, die ihre Immobilie aus eigener Tasche bezahlten. Die freiwillige Teilnahme schließlich war auch deshalb nicht sonderlich attraktiv, weil der Staat bei Naturkatastrophen in der Vergangenheit bewiesen hatte, dass er die Opfer nicht im Stich lassen würde. Die Ausgaben für Federal Disaster Relief stiegen von fünf Millionen Dollar 1950 auf fünf Milliarden Dollar im Jahr 1999 (Hood 2000). Als der Hurrikan Katrina 2005 auf die Golfküste traf, wurden die Unzulänglichkeiten des Systems vollends deutlich. In New Orleans hatte weniger als die Hälfte der versicherungsberechtigten Personen vom Angebot des NFIP, das seit 1979 von der neu gegründeten Federal Emergency Management Agency (FEMA) geleitet wird, Gebrauch gemacht.

Wie alle Versicherungen gegen Naturgefahren hatte das NFIP schließlich mit der Definition der Gefahrenart und der Abgrenzung von anderen, nicht versicherten Naturphänomenen zu kämpfen. „Was dem Einen ein frischer Wind ist, kann dem Anderen schon 
als Sturm erscheinen“, erkannte bereits der Verband Deutscher Privat-Feuerversicherungs-Gesellschaften (1898). Dass solche Differenzen keineswegs rein akademischer Natur waren, sondern sogar zu internationalen Verwicklungen führen konnten, hatte sich am deutlichsten nach dem Erdbeben 1906 in San Francisco gezeigt. Ob die Zerstörungen nämlich direkt vom Erdbeben oder indirekt von einem Feuer verursacht wurden, machte für die beteiligten Versicherer einen erheblichen Unterschied. Während viele Hausbesitzer gegen Brand abgesichert waren, fehlte in den meisten Policen eine Erdbebenklausel. Dies galt nicht zuletzt für die Verträge der auf dem US-Markt stark präsenten Münchner Rückversicherung (Steinberg 2000, 34, Röder 2006). Nach Katrina kam es zu ähnlichen „Interpretationsproblemen“, nur war die Frage nun nicht Erdbeben oder Feuer, sondern: Wind oder Wasser? Da die meisten Betroffenen im Rahmen der normalen Gebäudeversicherung gegen Wind, nicht aber gegen Fluten versichert waren, bestand ein großes Interesse daran, dem Sturm die Verursachung der Schäden zuzuschreiben - ein Konflikt, der zu etlichen, zumeist erfolglosen Prozessen führte (Grace/Klein 2007).

\section{Die Natur der Gefahr}

Mit der zunehmenden Integration von Flüssen und ihren Überschwemmungsgebieten in die Zweckrationalität wirtschaftlicher und sozialer Planung im Verlauf der letzten zwei Jahrhunderte ist nicht nur die ökonomische Leistungsfähigkeit der „Wasserstraßen“ immens gesteigert worden, sondern auch das desaströse Potenzial von Überschwemmungen. Dies gilt vor allem für die wirtschaftlichen und finanziellen Schäden, die solche Extremereignisse anrichten können, insbesondere dann, wenn durch Hochwasser ausgelöste ,infrastrukturelle Konflikte" die Schäden skalieren (Luebken 2007). Was Anfang des 19. Jahrhunderts noch ein primär lokales Ereignis war, kann heute im schlimmsten Fall globale Konsequenzen nach sich ziehen.

Das Instrument der Versicherung, das so erfolgreich in allen möglichen gesellschaftlichen Bereichen eingesetzt worden war, schien einen idealen Ansatz zu bieten, diese Problematik zu bewältigen. Rein privatwirtschaftliche Lösungen sind jedoch historisch immer wieder gescheitert, und wo sie heute existieren, sind sie eher kosmetischer Natur. So lange den wirklich gefährdeten Personen und Unternehmen Prämien abverlangt werden, die sie nicht bezahlen wollen oder können, so lange stellt die Überschwemmungsversicherung keine wirkliche Alternative zu staatlichen Hilfsleistungen dar. Mit Ulrich Beck kann man argumentieren, dass das „Fehlen eines angemessenen privaten Versicherungsschutzes (...) der institutionelle Indikator für den Übergang in die unkontrollierbare Risikogesellschaft der Zweiten Moderne" ist (2007, 239, Hervorhebung U.B.), in der das Versprechen des Versicherungsschutzes nur in Potemkinschen Dörfern aufrecht erhalten wird und die Risiken letztlich durch die unmittelbar von der Katastrophe Betroffenen zu tragen sind, die bestenfalls moralischen Anspruch auf staatliche Hilfe haben. ${ }^{9}$

Aus mehreren Gründen lässt sich die Versicherungslogik nur sehr bedingt auf Hochwasserschäden anwenden. Überschwemmungen sind, ebenso wie Terroranschläge und anders als etwa Autounfälle, ,katastrophale“ Risiken, d.h. die Eintrittswahrscheinlichkeit

9 Von einer Entsicherung oder Entkernung des Versicherungsschutzes, wie er seit einiger Zeit in den sozialen Sicherungssystemen zu beobachten ist, kann aber in diesem Fall schon deshalb nicht gesprochen werden, weil es den entsprechenden Schutz nie gegeben hat. 
ist gering, während das Ausmaß der Zerstörung potenziell sehr groß ist (Grace/Klein 2007). Da eine halbwegs zuverlässige Prognose unmöglich ist, bleibt ein Rest an Unsicherheit, der mit den gängigen Strategien des Risikomanagements nicht zu bewältigen ist. Dieses „Restrisiko“ muss, wenn eine Form des Versicherungsschutzes angestrebt wird, vom Staat als „ultimativem Risikomanager“ übernommen werden (Moss 2002), sei es durch Zwang zur Mitgliedschaft in der Risikogemeinschaft wie in der Schweiz oder durch ein eigenes Versicherungsprogramm wie in den USA. Was auf privatwirtschaftlichem Wege nicht erreicht werden kann - finanzieller Schutz vor den Folgen von Überschwemmungen und die Zahlung einer Prämie gemäß dem individuellen Grad der Gefährdung - soll auf diese Weise durch Regierungsintervention herbeigeführt werden.

Die obligatorische Elementarschadenversicherung wird zwar in der Schweiz erfolgreich praktiziert, ist allerdings an gewisse Voraussetzungen gebunden, die anderen Ortes selten erfüllt sind. Die Pflichtversicherung setzt nämlich eine als einigermaßen gleichmäBig wahrgenommene Gefährdungslage in allen Regionen des Staates voraus. In anderen Worten: Wenn eine Region das Gefühl hat, die Schadensbewältigung einer anderen Region über Gebühr zu subventionieren, wird eine Zwangsversicherung schwer durchzusetzen sein. So dürfte einer Versicherungsnehmerin im Sauerland schwer zu verdeutlichen sein, warum sie mit ihren Prämien zur Deckung von Lawinen- oder Bergsturzschäden im Süden und Sturmflutschäden an der Küste beitragen soll.

In den USA umging man dieses Problem durch die (zunächst) freiwillige Teilnahme an der staatlichen Versicherung und durch die Beschränkung auf Überschwemmungen. Doch auch dies wirft Gerechtigkeitsprobleme auf. Schon 1903 hielt die Neue Preußische Zeitung fest: „Aus politischen Gründen dürfte unseres Erachtens [eine staatliche Hochwasserversicherung] nicht angängig erscheinen, weil der Staat mit Rücksicht auf die Gleichberechtigung seiner Bürger nicht die Bewohner eines einzelnen Landstriches, der vorzugsweise Ueberschwemmungen ausgesetzt ist, durch eine mit Mitteln des Staates auszuführende Versicherung bevorzugen darf." Dem NFIP muss man zu gute halten, dass das Ziel einer Verlagerung des Risikomanagements vom Staat auf die Gefährdeten in „normalen“ Krisenjahren zumindest teilweise gelungen ist. Extreme hydrologische und meteorologische Ereignisse wie die Mississippi-Flut 1993 machen aber regelmäßig die Grenzen der Belastbarkeit deutlich. Zudem zeigt sich immer wieder, zuletzt an den Folgen von Hurrikan Katrina, dass gerade diejenigen, die Hilfe am nötigsten hätten, in der Regel nicht versichert sind.

Von den konkreten Schwierigkeiten der hier geschilderten Versuche, das „Verhalten“ der Natur rational, kalkulatorisch und damit auch finanziell in den Griff zu bekommen abgesehen, scheint darüber hinaus ein fundamentales Problem im Verhältnis von Natur und Gesellschaft durch. Von den Bemühungen des Unwetterausschusses bis zu Katrina werden immer wieder die Grenzen der Versicherbarkeit von Naturrisiken deutlich. Die natürliche Dynamik der Wasserläufe und der Ozeane, der Tiefdrucksysteme und der tropischen Stürme ließ sich (und lässt sich bis heute) eben nicht vollständig in die moderne Rationalität von Wetter- und Klimaprognosen, von mathematischen Modellen, Versicherungsstatistiken und Staatshaushalten übersetzen. Nicht nur Lewis Medlock, sondern auch die von ihm so verachteten Versicherungen mussten feststellen, dass Natur in diesem Sinne wortwörtlich unberechenbar ist. 


\section{Bibliographie}

American Insurance Association (1966) Brief an HUD, New York, 24. Juni 1966, National Archives and Records Administration (NA), College Park, Maryland/Record Group (RG) 311/Entry 3, B4.

Annalen des Deutschen Reichs (1899) Die Frage der Versicherung gegen Ueberschwemmungsschäden, 5: 408.

Annalen des gesamten Versicherungswesens (1899a) Versicherung gegen Unwetterschäden, 30 (2. November 1899).

Annalen des gesamten Versicherungswesens (1899b) Versicherung gegen Unwetterschäden, 30 (7. Dezember 1899).

Arnoldi (1931) Preußisches Ministerium für Landwirtschaft, Domänen und Forsten, an den Preußischen Innenminister, 16. Januar 1931, „Hochwasser- und Unwetterschädenversicherung “, Geheimes Staatarchiv Preußischer Kulturbesitz (GStA PK), I. HA, Rep. 77, Tit. 52, Nr. 124.

Arps, L. (1965) Auf sicheren Pfeilern: Deutsche Versicherungswirtschaft vor 1914. Göttingen: Vandenhoeck \& Ruprecht.

Barry, J. M. (1997) Rising Tide: The Great Mississippi Flood of 1927 and How It Changed America. New York: Simon \& Schuster.

Beck, U. (2007) Weltrisikogesellschaft: Auf der Suche nach der verlorenen Sicherheit. Frankfurt a.M.: Suhrkamp.

Beckstein, G. (2008) „Die Ruhe vor dem Sturm“, Rede anlässlich der Eröffnung des Symposiums der Versicherungskammer Bayern, München, 13. Februar. http://www.bayern.de/Anlage44017/Rede,13022008,Muenchen.pdf (29.07.08).

Böhm, H. R., et al. (1998) Anforderungen des vorsorgenden Hochwasserschutzes an Raumordnung, Landes-/Regionalplanung, Stadtplanung und die Umweltfachplanungen: Empfehlungen für die Weiterentwicklung. http://www.umweltdaten.de/publikationen/fpdf-1/1698.pdf (29.07.08).

Bonß, W. (1995) Vom Risiko. Unsicherheit und Ungewißheit in der Moderne. Hamburg: Hamburger Edition.

Deutsche Versicherungs-Zeitung (1900) Ein Epilog zur Unwetterversicherung, 28, 8. April 1900.

Ewald, F. (1989) Die Versicherungs-Gesellschaft. In: Kritische Justiz 22: 385-393.

Ewald, F. (1991) Insurance and Risk. In: Burchell, G./Gordon, C./Miller P. (ed.) The Foucault Effect. Chicago: Chicago University Press.

Fischer, M. (2004) Solidarität schafft Sicherheit. Die Kantonalen Gebäudeversicherungen als System. In: Pfister, C./Summermatter, S. (Hg.) Katastrophen und ihre Bewältigung: Perspektiven und Positionen. Bern, Stuttgart, Wien: Haupt.

Gerdes, V. (1963) Insuring the Flood Peril. In: The Journal of Insurance 30(4): 547-53.

Grace, M. F./Klein, R. W. (2007) Facing Mother Nature. In: Regulation 30(3): 28.

Grutzner, C. (1955) Flood Insurance: Pros and Cons. In: New York Times, 28. August.

Hood, J. (2000) Who Insures Against Floods, and Why. In: Consumers' Research Magazine 83(10): 16-20.

HUD (1966) Insurance and other Programs for Financial Assistance to Flood Victims, A Report from the Secretary of the Department of Housing and Urban Development (HUD) to the President, 8. August 1966, 20, NA/RG 311/Entry 2, B2. 
Hutchinson, J. (2000) Disasters and the International Order: Earthquakes, Humanitarians, and the Ciraolo Project, In: International History Review 22 (1/March 2000): 1-36.

Johner, W.H. (1958) Die Elementarschadenversicherung in der Schweiz. Der heutige Stand der Versicherung und die Möglichkeiten eines weiteren Ausbaus (InauguralDissertation, Universität Bern), Wintherthur: P.G. Keller.

Kalenberg, C. (1998) Zur Versicherbarkeit von Hochwasser- und Überschwemmungsschäden. Karlsruhe: Verlag der Versicherungswirtschaft.

King, R. (2005) Federal Flood Problem: The Repetitive Loss Problem. Congressional Research Service Report for Congress. http://fas.org/sgp/crs/misc/RL32972.pdf (30.07.08).

Kriebisch, H. (2001) Elementarschadensversicherungsdeckung auf der Grundlage des Zonierungssystems für Überschwemmung, Rückstau und Starkregen (ZÜRS). In: Umweltbundesamt Berlin/Institut für ökologische Raumentwicklung Dresden, Workshop Vorbeugender Hochwasserschutz auf kommunaler Ebene. http://www.umweltdaten.de/publikationen/fpdf-1/1964.pdf (30.07.08).

Landrat des Rheingaukreises an den Deutschen Versicherungs-Schutzverband (1957) Abschluß einer Versicherung gegen Hochwasser- und Eisschäden, Rüdesheim a. Rh., 5. September 1957; Hessisches Hauptstaatsarchiv Wiesbaden, Abt. 659, Nr. 858, Hochwasserschäden 1956 im Rheingaukreis.

Langbein, W. B. (1953) Flood Insurance. In: Land Economics 29(4): 323-330.

Lanz-Stauffer, H./Rommel, C. (1936) Elementarschäden und Versicherung. Bern: Selbstverlag des Rückversicherungsverbandes.

Luebken, U. (2007) ,Der große Brückentod‘: Überschwemmungen als infrastrukturelle Konflikte im 19. und 20. Jahrhundert. In: Saeculum. Jahrbuch für Universalgeschichte 58(1): 89-114.

Luhmann, N., Das Risiko der Versicherung gegen Gefahren. In: Soziale Welt 47: 273-83.

Makropoulos, M. (1990) Möglichkeitsbändigungen: Disziplin und Versicherung als Konzepte zur sozialen Sicherung von Kontingenz. In: Soziale Welt 41: 407-23.

Mohr, H. H. (1963) Grundlagen der Sturmversicherung, Karlsruhe: Verlag der Versicherungswirtschaft.

Moss, D. A. (2002) When All Else Fails: Government as the Ultimate Risk Manager, Cambridge, MA, London: Harvard University Press.

Münchner Rück (1927) Münchener Rückversicherungs-Gesellschaft an den Präsidenten des Reichsaufsichtsamtes für Privatversicherung (Abschrift), München, 20. September 1927, Bundesarchiv Berlin, R3101/17116.

Neue Preussische Zeitung (1903) Versicherung gegen Hochwasserschäden, 25. August 1903.

Oberholzner, F. (2006) Ein Novum der landwirtschaftlichen Risikovorsorge. Die Gründung der Bayerischen Landeshagelversicherungsanstalt 1884. In: Jahrbuch für Geschichte des ländlichen Raumes 3: 46-71.

Power, F. B./Shows E. W. (1979) A Status Report on the National Flood Insurance Program-Mid 1978. In: The Journal of Risk and Insurance 46 (2): $61-76$ (61).

Preußisches Innenministerium (1927a) Gesprächsprotokoll, Berlin, September 1927, „,Vermerk über die Besprechungen am 9./10. August in Bern, betr. die Fragen der Einführung einer Hochwasserversicherung “, GStA PK, I. HA, Rep. 77, Tit. 52, Nr. 124.

Preußisches Innenministerium (1927b) Vermerk über Besprechung am 14. September 1927, GStA PK, I. HA, Rep. 77, Tit. 52, Nr. 124. 
Preußisches Innenministerium (1930) Unbekannt an von Leyden und Simon, Berlin, März 1930, GStA PK, I. HA, Rep. 77, Tit. 52, Nr. 124.

Preußisches Statistisches Bureau (1900) Gutachten, o. D., zwischen August 1900 und März 1901. In: „Versicherung gegen Unwetterschäden“, Zeitschrift für Versicherungswesen, 28. August 1901.

Quante, P. (1926) Hochwasser und Überschwemmungsschäden, Preußisches Statistisches Landesamt, Berlin, 15. März 1926, GStA PK, I. HA, Rep. 77, Tit. 52, Nr. 124.

Reichsaufsichtsamt für Privatversicherung an Reichswirtschaftsministerium (1920) 19. März 1920, Bundesarchiv, R3101/17061.

Röder, T. J. (2006) Rechtsbildung im wirtschaftlichen „,Weltverkehr“. Das Erdbeben von San Francisco und die internationale Standardisierung von Vertragsbedingungen (1871-1914). Frankfurt a.M.: Vittorio Klostermann.

Rommel, C. (1950) Probleme der Hochwasser- und Überschwemmungsversicherung (Zeit- und Forschungsfragen der Versicherungswirtschaft, Heft 6). Weißenburg: Mathematischer Tabellen-Verlag.

Rotman Zelizer, V.A. (1979) Morals and Markets: The Development of Life Insurance in the United States. New York: Columbia University Press.

Schwarze, R./Wagner, G. G. (2004) In the Aftermath of Dresden: New Directions in German Flood Insurance. In: The Geneva Papers on Risk and Insurance 29(2): 154-68.

Steinberg, T. (2000) Acts of God: The Unnatural History of Natural Disaster in America. Oxford University Press, Oxford.

United States Senate, Banking and Currency Committee (1956) Federal Disaster Insurance. Staff Study, 84th Congress, 2nd Session, Report No. 1313, 09/01/56.

United States Task Force on Federal Flood Control Policy (1966) A Unified National Program for Managing Flood Losses. Washington, DC: U.S. Government Printing Office.

Unwetter-Ausschuss (1899) Sitzungsniederschrift, 24. März 1899, Bundesarchiv, R3101/17116.

Verband Deutscher Privat-Feuerversicherungs-Gesellschaften (1898) an von der Recke von der Horst, ,,Versicherung gegen Unwetterschäden “, Berlin, 6. Dezember 1898, Bundesarchiv, R3101/17116.

Verein der Industriellen des Regierungsbezirks Köln (1899) an Reichskanzler Hohenlohe, Köln, 28. März 1899, Bundesarchiv, R1501/117441.

Washington Post (2005) Editorial: Rethinking Flood Insurance, 21. September 2005.

White, G. F. (1945) Human Adjustment to Floods: A Geographical Approach to the Flood Problem in the United States. University of Chicago: Research Paper 29, Department of Geography.

Uwe Luebken, Historiker, ist wissenschaftlicher Mitarbeiter am Amerika-Institut der LMU München. Seine Forschungsschwerpunkte sind: US-amerikanische Geschichte, Umweltgeschichte, insbesondere Naturkatastrophen, Risikogeschichte und transnationale Geschichte.

E-Mail: uwe.luebken@1rz.uni-muenchen.de 